\title{
WITHDRAWAL BENEFITS UNDER A DEPENDENT DOUBLE DECREMENT MODEL
}

\author{
By Jacques F. CARriere \\ Dept. of Mathematical Sciences \\ University of Alberta
}

\begin{abstract}
This article presents an explicit formula for the value of a withdrawal benefit when the times of death and withdrawal are dependent. The derivation is based on an actuarial equivalence principle. As a special case, we show that in the fully continuous case, the withdrawal benefit is the reserve when the decrements are independent. We also present a definition of antiselection and prove that the withdrawal benefit will be smaller under antiselection.
\end{abstract}

\section{KeYwords}

Dependent decrement theory, withdrawal benefits, antiselection, the equivalence principle, varying life insurance.

\section{INTRODUCTION}

In some markets, like the United States, life insurance products have a withdrawal benefit when the policy is terminated. This article will examine the implications of dependent probabilities of withdrawal and death on withdrawal benefits for life insurance in discrete time. Specifically, we will give an explicit expression of the withdrawal benefit under a dependent decrement model thus allowing us to characterize the withdrawal benefit under antiselection.

In the book, Actuarial Mathematics (1986), the authors state that "if the withdrawal benefit in a double decrement model whole life insurance, fully continuous payment basis, is the reserve under the single decrement model whole life insurance, the premium and reserves under the double decrement model are equal to the premium and reserves under the single decrement model." This incredible result is not always true. The reason that the reserve is not always equal to the withdrawal benefit was given by D.R. Schuette (reported by Nesbitt (1964)), who found the withdrawal benefit is not the reserve in the discrete model because "the probability of withdrawal depends 
on the force of mortality." Thus, this article delves into the issue of dependent mortality and withdrawal in a discrete model. For an introduction to the mathematics of dependent decrement theory, consult Carriere (1994).

\section{The Single-Decrement Model}

In this section, we present the classical single-decrement model for life insurance pricing and reserving. Let $T_{d}$ denote the time and death for some life aged $x$. Next, let

$$
S^{d}(t)=\operatorname{Pr}\left(T_{d}>t\right), t \geq 0,
$$

be the survival function of $T_{d}$. Throughout the discussion we will assume that this survival function is absolutely continuous with a density denoted as $f^{d}(t)$ and a force of mortality equal to $\mu^{d}(t)=f^{d}(t) / S^{d}(t)$. Thus $S^{d}(t)=\exp \left\{-\int_{0}^{t} \mu^{d}(z) d z\right\}$. Now consider the probability that the life survived to time $t+s$ given that it survived to time $t$. This survival function is denoted as $p_{t}^{d}$ and it is equal to:

$$
s p_{t}^{d}=\operatorname{Pr}\left(T_{d}>t+s \mid T_{d}>t\right)=\frac{S^{d}(t+s)}{S^{d}(t)}=\exp \left\{-\int_{t}^{t+s} \mu^{d}(z) d z\right\} .
$$

It will be convenient to define $T_{d}(t)$ as the random variable induced by ${ }_{s} p_{t}^{d}$ so that ${ }_{s} p_{t}^{d}=\operatorname{Pr}\left(T_{d}(t)>s\right)$. Note that $T_{d}=T_{d}(0)$ and ${ }_{t} p_{0}^{d}=S^{d}(t)$. Moreover, if $T_{d}>t$ then $T_{d}(t)=T_{d}-t$, otherwise $T_{d}(t)$ is undefined. It is instructive to note that if the expectation $E\left[g\left(T_{d}(t)\right)\right]$ exists for some function $g(s)$, then

$$
E\left[g\left(T_{d}(t)\right)\right]=E\left[g\left(T_{d}-t\right) \mid T_{d}>t\right] .
$$

This last fact will be used repeatedly.

Usually we will assume that premiums and death benefits are paid at the discrete times $t=k / m$ where $k=0,1,2, \ldots$ and $m>0$. Therefore, it is convenient to define the discrete random variable

$$
K_{d}^{m}=\frac{\left\lfloor m T_{d}\right\rfloor}{m},
$$

where $\lfloor$.$\rfloor is the floor function. In other words, \lfloor y\rfloor$ is the integer part of $y$. Thus, if $y \geq 0$ then $\lfloor y\rfloor=k$ if and only if $k \leq y<k+1$. Note that $T_{d}$ is simply equal to $K_{d}^{m}$ when $m=\infty$ and so any discussion about the continuous model is subsumed within the discrete model.

In this article, we assume that the life insurance has a varying death benefit equal to $b(t)$, if death occurs at time $t$. Typically, $b(t)=1$ for all $t$. 
Next, let

$$
v(t)=\exp \left\{-\int_{0}^{t} \delta_{z} d z\right\}
$$

denote the interest discount function. Traditionally, actuaries have assumed that $\delta_{z}=\delta$ is constant thus implying that $v(t)=e^{-\delta t}$. Using the equivalence principle and the functions $v(t)$ and $b(t)$, we define the net single premium at time $t$ for the future benefits from the life insurance as:

$$
\begin{aligned}
A_{d}^{m}(t) & =E\left[b\left(K_{d}^{m}+1 / m\right) v\left(K_{d}^{m}+1 / m\right) / v(t) \mid T_{d}>t\right] \\
& =E\left[b\left(\left\lfloor m T_{d}+1\right\rfloor / m\right) v\left(\left\lfloor m T_{d}+1\right\rfloor / m\right) / v(t) \mid T_{d}>t\right] \\
& =E\left[b\left(\left\lfloor m\left(T_{d}(t)+t\right)+1\right\rfloor / m\right) v\left(\left\lfloor m\left(T_{d}(t)+t\right)+1\right\rfloor / m\right) / v(t)\right] .
\end{aligned}
$$

Note that the last equality in equation (6) follows from equation (3). Now, let us focus on the valuation of the premium payments. Assume that payments of $1 / m$ are made at the times $t=0,1 / m, 2 / m, \ldots$. Then the present value at time $t=0$ of all the payments made in the period $[r, s)$ will be denoted as $a[r, s)$ and calculated as:

$$
a[r, s)=\frac{1}{m} \sum_{k=0}^{\infty} v(k / m) 1(r \leq(k / m)<s) .
$$

In this definition, $1(e)$ is an indicator function that is equal to 1 if the event $e$ is true and 0 , otherwise. It is instructive, to verify that $a[r, s]=a[0, s)-a[0, r)$. Using this annuity-certain formula and the equivalence principle, we find that the net single premium for the future payments from the life annuity at time $t$ is:

$$
\begin{aligned}
a_{d}^{m}(t) & =E\left[a\left[t, K_{d}^{m}+1 / m\right) / v(t) \mid T_{d}>t\right] \\
& =E\left[a\left[t,\left\lfloor m T_{d}+1\right\rfloor / m\right) / v(t) \mid T_{d}>t\right] \\
& =E\left[a\left[t,\left\lfloor m\left(T_{d}(t)+t\right)+1\right\rfloor / m / v(t)\right] .\right.
\end{aligned}
$$

Under the single decrement model, the net level premium for the life insurance is denoted as $P_{d}^{m}$ and it is equal to:

$$
P_{d}^{m}=A_{d}^{m}(0) / a_{d}^{m}(0),
$$

under the equivalence principle. Thus, we can define the link function as:

$$
\mathcal{L}(r, s)=b(s) v(s) / v(r)-P_{d}^{m} a[r, s) / v(r),
$$

This link function will be useful when the withdrawal benefit is derived for the double-decrement model in the next section. This link function can also be used to define the prospective loss at time $t$, which is:

$$
L^{m}(t)=\mathcal{L}\left(t, K_{d}^{m}+1 / m\right) \text {. }
$$


Note that $E\left[L^{m}(0)\right]=0$. Finally, we find that the prospective reserve at any time $t$ is:

$$
\begin{aligned}
V^{m}(t) & =E\left[L^{m}(t) \mid T_{d}>t\right]=E\left[\mathcal{L}\left(t,\left\lfloor m T_{d}+1\right\rfloor / m\right) \mid T_{d}>t\right] \\
& =E\left[\mathcal{L}\left(t,\left\lfloor m\left(T_{d}(t)+t\right)+1\right\rfloor / m\right)\right]=A_{d}^{m}(t)-P_{d}^{m} a_{d}^{m}(t) .
\end{aligned}
$$

Note that the random variable $\left\lfloor m\left(T_{d}(t)+t\right)+1\right\rfloor / m$ has a central role. Thus it will be convenient to define

$$
\mathcal{K}_{d}^{m}(t)=\left\lfloor m\left(T_{d}(t)+t\right)+1\right\rfloor / m .
$$

With this notation, we can write

$$
\begin{aligned}
A_{d}^{m}(t) & =E\left[b\left(\mathcal{K}_{d}^{m}(t)\right) v\left(\mathcal{K}_{d}^{m}(t)\right) / v(t)\right], \\
a_{d}^{m}(t) & =E\left[a\left[t, \mathcal{K}_{d}^{m}(t)\right) / v(t)\right], \\
V^{m}(t) & =E\left[\mathcal{L}\left(t, \mathcal{K}_{d}^{m}(t)\right)\right] .
\end{aligned}
$$

Let $S^{d}(s \mid t, m)$ denote the survival function of $\mathcal{K}_{d}^{m}(t)$. Let us derive this function. Consider the fact that if $y \geq 0$ and $x \geq 0$, then $\lfloor y\rfloor+1>x$ if and only if $y \geq\left\lfloor x \mid\right.$. Using this result we find that $\mathcal{K}_{d}^{m}(t)>s$ if and only if $T_{d}(t) \geq(\lfloor m s\rfloor / m)-t$. Therefore,

$$
S^{d}(s \mid t, m)=\exp \left\{-\int_{t}^{\lfloor m s\rfloor / m} \mu^{d}(z) d z\right\} .
$$

\section{The Double-Decrement Model}

In this section, we present the probabilistic structure for a dependent doubledecrement model. This will allow us to derive an expression for the withdrawal benefit, $W_{d \mid w}^{m}(t)$, that represents the benefit that is returned to the policyholder at time $\lfloor m t+1\rfloor / m$ when withdrawal occurs at time $t$.

Let $T_{w}$ denote the time at withdrawal from a life insurance contract for a life aged $x$, where $f^{w}(t)$ is the density, $S^{w}(t)=\int_{t}^{\infty} f^{w}(z) d z$ is the survival function and $\mu^{w}(t)=f^{w}(t) / S^{w}(t)$ is the force. We will find it useful to define the discrete random variable

$$
K_{w}^{m}=\frac{\left\lfloor m T_{w}\right\rfloor}{m} .
$$

Generally, we assume that $T_{d}$ and $T_{w}$ are not stochastically independent. Therefore, let us consider the conditional density of $T_{d}$ given that $T_{w}=t$, which is denoted as $f^{d \mid w}\left(t_{d} \mid t\right)$. Also, let $S^{d \mid w^{\prime}}\left(t_{d} \mid t\right)=\int_{t_{d}}^{\infty} f^{d \mid w^{\prime}}(z \mid t) d z$ denote the 
conditional survival function of $T_{d}$ given that $T_{w}=t$. Hence, the conditional force of mortality is

$$
\mu^{d \mid w^{w}}\left(t_{d} \mid t\right)=\frac{f^{d \mid w}\left(t_{d} \mid t\right)}{S^{d \mid w}\left(t_{d} \mid t\right)}
$$

Thus $S^{d \mid w}\left(t_{d} \mid t\right)=\exp \left\{-\int_{0}^{t_{d}} \mu^{d \mid w}(z \mid t) d z\right\}$. In the case of independence, we get $f^{d \mid w}\left(t_{d} \mid t\right)=f^{d}\left(t_{d}\right), S^{\left.d\right|_{w} ^{w}}\left(t_{d} \mid t\right)=S^{d}\left(t_{d}\right)$, and $\mu^{d \mid w}\left(t_{d} \mid t\right)=\mu^{d}\left(t_{d}\right)$. It is important to note that the ensuing discussion and results assume that we know the density $f^{d \mid w}\left(t_{d} \mid t\right)$. However, estimating this density is not a trivial exercise because we can only observe the minimum of the random variables $T_{d}$ and $T_{w}$.

Now consider the probability that the life survived to time $t+s$ given that it survived to time $t$ and withdrawal occurred at time $t$. This survival function is denoted as $s_{t}^{d \mid w^{\prime}}$ and it is equal to:

$$
\begin{aligned}
{ }_{s} p_{t}^{d \mid w} & =\operatorname{Pr}\left(T_{d}>t+s \mid T_{d}>t, T_{w}=t\right)=\frac{\operatorname{Pr}\left(T_{d}>t+s, T_{d}>t \mid T_{w}=t\right)}{\operatorname{Pr}\left(T_{d}>t \mid T_{w}=t\right)} \\
& =\frac{S^{d \mid w}(t+s \mid t)}{S^{d \mid w}(t \mid t)}=\exp \left\{-\int_{t}^{t+s} \mu^{d \mid w}(z \mid t) d z\right\} .
\end{aligned}
$$

It will be convenient to define $T_{d \mid w}(t)$ as the random variable induced by ${ }_{s} p_{t}^{d \mid w}$ so that $s_{t}^{d \mid w}=\operatorname{Pr}\left(T_{d \mid w}(t)>s\right)$. Note that $p_{0}^{d}=S^{d \mid w}(t \mid 0)$. We let $T_{d \mid w}(0)=T_{d \mid w}$. It is instructive to note that if the expectation $E\left[g\left(T_{d \mid w}(t)\right)\right]$ exists for some function $g(s)$, then

$$
E\left[g\left(T_{d \mid w}(t)\right)\right]=E\left[g\left(T_{d}-t\right) \mid T_{d}>t, T_{w}=t\right] .
$$

This last fact will be used repeatedly. In the definition of the withdrawal benefit, the random variable $\left[m\left(T_{d \mid w}(t)+t\right)+1\right\rfloor / m$ has a central role. Thus it will be convenient to define

$$
\mathcal{K}_{d \mid w}^{m}(t)=\left\lfloor m\left(T_{d \mid w}(t)+t\right)+1\right\rfloor / m .
$$

With this notation, we can write

$$
\begin{aligned}
A_{d \mid w}^{m}(t) & =E\left[b\left(\mathcal{K}_{d \mid w}^{m}(t) v\left(\mathcal{K}_{d \mid w}^{m}(t)\right) / v(t)\right],\right. \\
a_{d \mid \psi^{\prime}}^{m}(t) & =E\left[a\left[t, \mathcal{K}_{d \mid w}^{m}(t)\right) / v(t)\right] .
\end{aligned}
$$

Let $S^{d \mid w}(s \mid t, m)$ denote the survival function of $K_{d \mid w}^{m}(t)$. Note that $K_{d \mid w}^{m}(t)>s$ if and only if $T_{d \mid w}(t) \geq\lfloor m s\rfloor / m-t$. Therefore,

$$
S^{d \mid w}(s \mid t, m)=\exp \left\{-\int_{t}^{\lfloor m s\rfloor / m} \mu^{d \mid w}(z) d z\right\} .
$$

We are now ready to state our first theorem. 
Theorem 3.1. Let $\mathcal{L}(t, s)$ be the link function. If the equivalence principle holds, then under a double-decrement model where the premiums are equal to $P_{d}^{m}$, the withdrawal benefit function is:

$$
\begin{aligned}
W_{d \mid w}^{m}(t) & =E\left[\mathcal{L}\left(\lfloor m t+1\rfloor / m, \mathcal{K}_{d \mid w}^{m}(t)\right)\right] \\
& =E\left[\mathcal{L}\left(\lfloor m t+1\rfloor / m,\left\lfloor m\left(T_{d \mid w}(t)+t\right)+1\right\rfloor / m\right)\right] \\
& =E\left[\mathcal{L}\left(\lfloor m t+1\rfloor / m,\left\lfloor m T_{d}+1\right\rfloor / m\right) \mid T_{d}>t, T_{w}=t\right] \\
& =E\left[\mathcal{L}\left(\lfloor m t+1\rfloor / m, K_{d}^{m}+1 / m\right) \mid T_{d}>t, T_{w}=t\right] .
\end{aligned}
$$

Proof. If $W_{d \mid w}^{m}(t)$ is the withdrawal benefit, then under the equivalence principle,

$$
\begin{aligned}
& P_{d}^{m} E\left[a\left[0, K_{d}^{m}+1 / m\right) 1\left(T_{d} \leq T_{w}\right)+a\left[0, K_{w}^{m}+1 / m\right) 1\left(T_{d}>T_{w}\right)\right]= \\
& E\left[b\left(K_{d}^{m}+1 / m\right) v\left(K_{d}^{m}+1 / m\right) 1\left(T_{d} \leq T_{w}\right)+W_{d \mid w}^{m}\left(T_{w}\right) v\left(K_{w}^{m}+1 / m\right) 1\left(T_{d}>T_{w^{\prime}}\right)\right] .
\end{aligned}
$$

Therefore,

$$
\begin{aligned}
& P_{d}^{m} E\left[a\left[0, K_{d}^{m}+1 / m\right)\right]+ \\
& P_{d}^{m} E\left[\left[a\left[0, K_{w}^{m}+1 / m\right)-a\left[0, K_{d}^{m}+1 / m\right)\right] 1\left(T_{d}>T_{w}\right)\right]= \\
& E\left[b\left(K_{d}^{m}+1 / m\right) v\left(K_{d}^{m}+1 / m\right)\right]+ \\
& E\left[\left[W_{d \mid w}^{m}\left(T_{w}\right) v\left(K_{w}^{m}+1 / m\right)-b\left(K_{d}^{m}+1 / m\right) v\left(K_{d}^{m}+1 / m\right)\right] 1\left(T_{d}>T_{w}\right)\right] .
\end{aligned}
$$

But

$$
P_{d}^{m} \times E\left[a\left[0, K_{d}^{m}+1 / m\right)\right]=E\left[b\left(K_{d}^{m}+1 / m\right) v\left(K_{d}^{m}+1 / m\right)\right] .
$$

For simplicity, let $Y=1\left(T_{d}>T_{w}\right) v\left(K_{w}^{m}+1 / m\right)$. Then

$$
\begin{aligned}
& E\left[Y W_{d \mid w}^{m}\left(T_{w}\right)\right]= \\
& E\left[Y b\left(K_{d}^{m}+1 / m\right) v\left(K_{d}^{m}+1 / m\right) / v\left(K_{w}^{m}+1 / m\right)\right]- \\
& E\left[Y P_{d}^{m}\left(a\left[0, K_{d}^{m}+1 / m\right)-a\left[0, K_{w}^{m}+1 / m\right)\right) / v\left(K_{w}^{m}+1 / m\right)\right] .
\end{aligned}
$$

Note that $a\left[0, K_{d}^{m}+1 / m\right)-a\left[0, K_{w}^{m}+1 / m\right)=a\left[K_{w}^{m}+1 / m, K_{d}^{m}+1 / m\right)$, hence the right-hand side of the last equation is equal to

$$
\begin{aligned}
& E\left[Y \mathcal{L}\left(K_{w}^{m}+1 / m, K_{d}^{m}+1 / m\right)\right]=E\left[Y E\left[\mathcal{L}\left(K_{w}^{m}+1 / m, K_{d}^{m}+1 / m\right) \mid T_{d}>T_{w}, T_{w}\right]\right]= \\
& \left.\left.E\left[Y E\left[\mathcal{L}\lfloor m t+1\rfloor / m, K_{d}^{m}+1 / m\right) \mid T_{d}>t, T_{w}=t\right]\right|_{t=T_{w}}\right]=E\left[Y W_{d \mid w}^{m}\left(T_{w}\right)\right] .
\end{aligned}
$$

Hence, the result is proved.

Now, let us compare the withdrawal benefit $W_{d \mid w}^{m}(t)$, as defined in equation (23), with the reserve formula $V^{m}(t)$, shown in equation (12). Clearly, they are different, even when $T_{d}$ and $T_{w}$ are stochastically independent. In the case of independence, $T_{d}(t)$ and $T_{d \mid w}(t)$ are identically 
distributed. Let $W_{d}^{m}(t)$ denote the withdrawal benefit under the independent decrement model, then

$$
W_{d}^{m}(t)=E\left[\mathcal{L}\left(\lfloor m t+1\rfloor / m, \mathcal{K}_{d}^{m}(t)\right)\right] .
$$

Thus

$$
\lim _{m \rightarrow \infty} V^{m}(t)=\lim _{m \rightarrow \infty} W_{d \mid w}^{m}(t)=E\left[\mathcal{L}\left(t, T_{d}(t)+t\right)\right] .
$$

In other words, the withdrawal benefit is equal to the reserve in the continuous model, thus confirming a well-known fact.

\section{Withdrawal Benefits under Antiselection}

In this section, we give a definition of antiselection and we show that the withdrawal benefit under antiselection is smaller than the benefit under the single-decrement model, as expected. We are now ready to give a definition of antiselection. We say that life insurance is subject to antiselection at withdrawal, if

$$
\mu^{d \mid w}\left(t_{d} \mid t_{w}\right)<\mu^{d}\left(t_{d}\right) \forall t_{d} \geq t_{w} .
$$

If we reverse this inequality, then we have antiselection for life annuities. Using our definition of antiselection, we immediately find that

$$
S^{d \mid w}(s \mid t, m) \geq S^{d}(s \mid t, m) .
$$

for alls $s \geq 0$.

First, we discuss the implications of antiselection to the valuation of the life insurance. Assume that $g(s)=b(s) v(s)$ is an absolutely continuous function with $g^{\prime}(s) \leq 0$ so that

$$
g(s)=g(0)+\int_{0}^{s} g^{\prime}(z) d z .
$$

Actually, this is a weak assumption because the assumption is obviously true when $b(s)=1$ and $v(s)=\exp (-\delta s)$.

Lemma 4.1. Suppose that $g(s)=b(s) v(s)$ for $s \geq 0$ is absolutely continuous and $g^{\prime}(s) \leq 0$. If $g(s)$ is integrable with respect to the cumulative distribution functions $1-S^{d \mid w}(s \mid t, m)$ and $1-S^{d}(s \mid t, m)$, then under the antiselection condition $\mu^{d \mid w}\left(t_{d} \mid t_{w}\right)<\mu^{d}\left(t_{d}\right)$ and the equivalence principle, we get

$$
A_{d \mid w}^{m}(t) \leq A_{d}^{m}(t) .
$$


Proof. First, note that

$$
\begin{aligned}
v(t) A_{d}^{m}(t) & =E\left[b\left(\mathcal{K}_{d}^{m}(t)\right) v\left(\mathcal{K}_{d}^{m}(t)\right)\right] \\
& =\int_{[0, \infty)} g(s) d\left(1-S^{d}(s \mid t, m)\right) \\
& =\int_{[0, \infty)}\left[g(0)+\int_{0}^{s} g^{\prime}(z) d z\right] d\left(1-S^{d}(s \mid t, m)\right) \\
& =g(0)+\int_{0}^{\infty} \int_{(z, \infty)} g^{\prime}(z) d\left(1-S^{d}(s \mid t, m)\right) d z \\
& =g(0)+\int_{0}^{\infty} g^{\prime}(z) S^{d}(z \mid t, m) d z
\end{aligned}
$$

Next, under antiselection $S^{d \mid w}(z \mid t, m) \geq S^{d}(z \mid t, m)$ and so $g^{\prime}(z) S^{d \mid w}(z \mid t, m) \leq$ $g^{\prime}(z) S^{d}(z \mid t, m)$. Integrating both sides of the inequality yields the result.

Next, we present a lemma on the implications of antiselection to the valuation of life annuities.

Lemma 4.2. Under the equivalence principle and the antiselection condition $\mu^{d \mid w}\left(t_{d} \mid t_{w}\right)<\mu^{d}\left(t_{d}\right)$, we get

$$
a_{d \mid w}^{m}(t) \geq a_{d}^{m}(t)
$$

Proof. First, note that

$$
\begin{aligned}
v(t) a_{d}^{m}(t) & =E\left[a\left[t, \mathcal{K}_{d}^{m}(t)\right)\right] \\
& =E\left[\frac{1}{m} \sum_{k=0}^{\infty} v(k / m) 1\left(t \leq k / m<\mathcal{K}_{d}^{m}(t)\right)\right] \\
& =\frac{1}{m} \sum_{k=0}^{\infty} v(k / m) 1(t \leq k / m) E\left[1\left(k / m<\mathcal{K}_{d}^{m}(t)\right)\right] \\
& =\frac{1}{m} \sum_{k=0}^{\infty} v(k / m) 1(t \leq k / m) S^{d}(k / m \mid t, m) .
\end{aligned}
$$

But under antiselection $S^{d \mid w}(s \mid t, m) \geq S^{d}(s \mid t, m)$. Summing both sides of the inequality yields the result.

Applying Lemma 4.1 and 4.2 , we immediately find that under antiselection the withdrawal benefit under the classical independent decrement model is too large. We summarize this result with the following theorem. 
Theorem 4.3. Under the conditions in Lemma 4.1 and 4.2, we get

$$
W_{d \mid w^{\prime}}^{m}(t) \leq W_{d}^{m}(t)
$$

\section{REFERENCES}

Bowers, N.L., Gerber, H.U., Hickman, J.C., Jones, D.A. and Nesbitt, C.J. (1986). Actuarial Mathematics. Society of Actuaries: Schaumburg, Illinois.

CARriere, J.F. (1994). "Dependent decrement theory." Transactions: Society of Actuaries, XLVI, 45-73.

NesbitT, C.J. (1964). Discussion of "A Statistical Approach to Premiums and Reserves in Multiple Decrement Theory." Transactions: Society of Actuaries, XVI, Part I, 149-153.

Professor Jacques Carriere

Dept. of Mathematical Sciences

University of Alberta

Edmonton, Alberta

Canada T6G 2GI 